\title{
ESTUDIOS LIMNOLÓGICOS EN LAGOS Y LAGUNAS DEL PARQUE NACIONAL TORRES DEL PAINE (51 S, CHILE).
}

\author{
LIMNOLOGICAL STUDIES IN LAKES AND PONDS OF TORRES \\ DEL PAINE NATIONAL PARK $\left(51^{\circ} \mathrm{S}, \mathrm{CHILE}\right)$.
}

Patricio De los Ríos ${ }^{1} \&$ Doris Soto ${ }^{2}$

\begin{abstract}
RESUMEN
La Patagonia de Chile posee muchos ecosistemas acuáticos sin contaminación, dentro de estos ecosistemas hay muchos lagos y lagunas prístinos. En este estudio, se analizó información colectada entre los años 1989 y 1990 (sin publicar y sin publicar) sobre lagos y lagunas del Parque Nacional Torres del Paine, un área sin contaminar en el sur de Chile (51 $\mathrm{S})$. Se aplicó un análisis de componentes principales considerando parámetros químicos, riqueza de especies zooplanctónicas y biomasa. Los resultados denotaron que las concentraciones de cationes ( $\mathrm{Ca}, \mathrm{Na}$ y $\mathrm{Mg}$ ), cloruros, seston y clorofila, junto con biomasa de zooplancton y riqueza de especies zooplanctónicas fueron las principales variables que contribuyeron al modelo obtenido. Según este modelo, se observaron los siguientes grupos: un primer grupo conformado con lagos oligotróficos con baja biomasa de zooplancton y baja riqueza de especies; un segundo grupo conformado por pequeños lagos mesotróficos con conductividad moderada, biomasa y riqueza de especies zooplanctónicas moderadas, dentro de este grupo hubo además pequeñas lagunas con altas concentraciones de nutrientes, clorofila y alta biomasa y riqueza de especies zooplanctónicas. Se aplicó un modelo nulo para co-ocurrencia de especies, que mostró que las asociaciones de especies no son aleatorias, lo que se demostró en dos de las tres simulaciones, lo cual concordaría con los resultados del análisis de componentes principales. Estos resultados revelarían que la oligotrofía y las bajas concentraciones de iones serían los principales factores reguladores de la estructura de la comunidad zooplanctónica, y probablemente la presencia de peces, lo cual explicaría los patrones similares observados para lagos de la Patagonia de Argentina y Chile.
\end{abstract}

Palabras clave: lagos, lagunas, clorofila, nutrientes, iones, zooplancton.

1 Universidad Católica de Temuco, Escuela de Ciencias Ambientales, Casilla 15-D, Temuco, Chile. prios@uct.cl

2 Senior Fisheries Officer Inland Water Resources and Aquaculture Service (FIRI), Fisheries Department, FAO of UN, Via delle Terme di Caracalla, I-00100, Roma, Italia. 


\section{ABSTRACT}

Chilean Patagonia has numerous unpolluted terrestrial and aquatic ecosystems, containing many unpolluted and pristine lakes and ponds. Published and unpublished information collected between 1989 and 1990 in lakes and ponds within Torres del Paine National Park, an unpolluted and pristine protected area in Southern Chile $\left(51^{\circ} \mathrm{S}\right)$, was analysed in this study. A principal component analysis (PCA) was applied using mainly chemical parameters, zooplankton species richness and biomass. It was found that the cations ( $\mathrm{Ca}, \mathrm{Na}, \mathrm{Mg}$ ) chloride, seston and chlorophyll concentrations together with zooplankton biomass and zooplankton species richness were the main contributing variables to the derived model. According to this model, the following groups were observed: $1^{\text {st }}$ group- deep oligotrophic lakes with low zooplankton biomass and low species richness; $2^{\text {nd }}$ group - small mesotrophic lakes and ponds with moderate conductivity, zooplankton biomass and species richness. This latter group included small ponds with high nutrient and chlorophyll concentrations. A null model for species co-ocurrence was applied. The results in two of three simulations denoted that species associations are not random, which would agree with the PCA results. These results showed that oligotrophy and low ionic concentrations, and probably fish presence, are important regulatory factors in zooplankton community structure. These explain the similar patterns observed between Argentinean and Chilean Patagonian lakes.

Key words: lakes, ponds, chlorophyll, nutrients, ions, zooplankton.

\section{INTRODUCCIÓN}

La Patagonia chilena $\left(39-53^{\circ} \mathrm{S}\right)$ es una zona poco contaminada, con bosques lluviosos y algunas praderas (Soto \& Zuñiga 1991, Soto et al. 1994). Esta zona tiene numerosos lagos oligotróficos profundos caracterizados por su baja concentración de iones, bajo número de especies en general, y estructura zooplanctónica con alto predominio de copépodos calanoideos (Soto \& Zúñiga 1991). En el sur de la Patagonia $\left(51^{\circ} \mathrm{S}\right)$ hay numerosos lagos y lagunas mesotróficas, con concentración de iones, con alta biomasa zooplanctónica y alto número de especies de crustáceos zooplanctónicos (Soto et al. 1994). Estas observaciones son similares a las observadas para lagos y lagunas de la Patagonia de Argentina (Modenutti et al. 1998). Estos patrones en los ensambles zooplanctónicos son marcadamente diferentes a las descripciones realizadas para lagos del hemisferio norte, donde hay alta riqueza de especies y alta dominancia de cladóceros de la familia Daphnidae (Soto \& Zúñiga 1991, Gillooly \& Dodson 2000).

La causa del bajo número de especies se debe a la oligotrofia de los sitios (Soto \& Zúñiga 1991, Woelfl 2007), porque según la ecología de comunidades existe una relación directa entre productividad del ecosistema y la riqueza de especies
(Jaksic 2001). Otro factor importante que regula el número de especies es la concentración de iones, ya que ambas están inversamente relacionadas (Williams 1998). Sobre esta base, ambos factores, o sea estatus trófico y concentración de iones serían los reguladores principales de las comunidades fitoplanctónicas y zooplanctónicas en lagos del sur de la Patagonia chilena (Soto et al. 1994). El presente estudio se realizó en el Parque Nacional Torres del Paine desde 1989 a 1990. El objetivo fue analizar los parámetros químicos y tróficos, biomasa zooplanctónica y riqueza de especies zooplanctónicas en lagos prístinos y lagunas del parque. De igual modo, se aplicará un modelo nulo de co-ocurrencia de especies con el fin de determinar la presencia o ausencia de factores reguladores de las asociaciones de especies zooplanctónicas en los sitios estudiados.

\section{MATERIAL Y MÉTODOS}

El Parque Nacional Torres del Paine está localizado en la zona sur de Chile (5055' S, $\left.73^{\circ} 05^{\prime} \mathrm{W}\right)$, tiene aproximadamente 242.240 hectáreas y fue declarado Reserva de la Biósfera por la UNESCO. Dentro del parque hay una alta diversidad de paisajes, glaciares, valles, campos de nieve, lagos y lagunas (Soto et al. 1994). El clima dentro del parque es subpolar, frío y seco, con menos de $700 \mathrm{~mm}$ de 
precipitaciones anuales. En noviembre y diciembre el parque está expuesto a fuertes vientos de 100 $\mathrm{km} / \mathrm{hr}$ (Campos et al. 1994a,b; Soto et al. 1994). Dentro del parque hay una serie de lagos profundos y pequeñas lagunas poco profundas con diferentes concentraciones iónicas y estatus trófico (Soto et al. 1994), las características geográficas están especificadas en la figura 1 y tabla 1 .

Los datos de estatus trófico, superficie, profundidad y conductividad fueron obtenidos a partir de Soto et al. (1994). De igual modo se consideró la riqueza de especies de crustáceos zooplanctónicos (CZSR) y se consideró la identificación a nivel de especies (Soto \& De los Ríos 2006). Las muestras para nutrientes (Nitrato: $\mathrm{NO}_{3}^{-}$, Nitrogeno Total: TN, Amonio: $\mathrm{NH}_{4}^{+}$, Fósforo Total: TP, Fósforo Reactivo Soluble: $\mathrm{PO}_{4}{ }^{3-}$ ); aniones (Cloruro: $\mathrm{Cl}$, y Sulfato: $\mathrm{SO}_{4}{ }^{2-}$ ); cationes (Calcio: $\mathrm{Ca}^{2+}$, Sodio: $\mathrm{Na}^{+}$, Potasio: $\mathrm{K}^{+} \mathrm{y}$ Magnesio: $\mathrm{Mg}^{2+}$ ); seston (Seston Total: S. T., Seston Inorgánico: S.I. y Seston Orgánico S.O.); Dureza Total (TH) y Dureza Alemana (GH), Clorofila a (Chl a) y silicato $\left(\mathrm{SiO}_{2}\right)$ fueron colectadas en la superficie y cuantificadas según las metodologías de Campos et al. (1994a,b). Según (Campos et al. 1994a,b), el nitrato se determinó de manera colorimétrica por el método del salicilato de sodio con sal Seigenette. El fósforo total se midió con el método ácido ascórbico y antimonil-tartrato de potasio después de una digestión con ácido sulfúrico y peróxido de hidrógeno al 30 \%. El Nitrógeno Total se determinó con el método Kjeldahl, el Fósforo Reactivo Soluble se determinó por medio de la metodología del ácido ascórbico y antimonil tartrato de potasio, el pH se midió por medio de un sensor portátil compuesto de electrodos de gas, el $\mathrm{CO}_{2}$ se midió por medio del método titrimétrico con hidróxido de sodio al 0,002 M y fenoftaleína; la alcalinidad se midió por medio de titulación con ácido clorhídrico al 0,025 N con verde bromocresol metilado como indicador. $\mathrm{El}$ Seston Total (S.T.), Seston Orgánico (S.O.) y Seston Inorgánico (S.I.) se determinó por medio de filtración de un litro de agua a través de papel de filtro de fibra de vidrio que fue previamente incinerado en horno (Campos et al. 1994a,b). Todos estos métodos han sido frecuentemente utilizados en estudios limnológicos en lagos chilenos (Soto 2002).

Las muestras se colectaron durante septiembre de 1988, enero, mayo y octubre de 1989 y enero de 1990. Se colectó una muestra por periodo, sumando

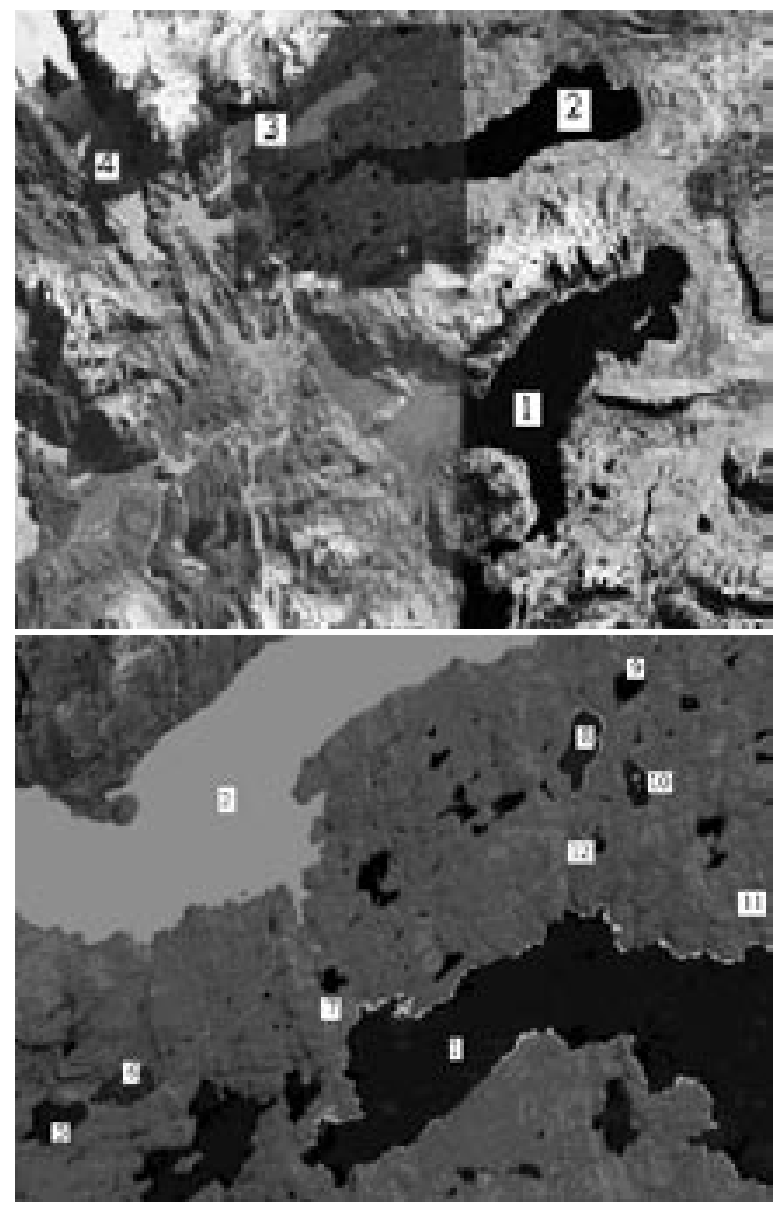

Fig. 1. Mapa de los sitios estudiados (obtenido a partir de Google Earth). Lagos: 1) Del Toro; 2) Sarmiento; 3) Norsdenkjold; 4) Pehoe; Lagos chicos: 5) Melliza Oeste; 6) Melliza Este; Lagunas: 7) Paso de la Muerte; 8) Larga;

9) Redonda; 10) Cisnes; 11) Jovito; 12) Juncos.

un total de cinco muestras porque sólo en estos periodos están las condiciones óptimas para el trabajo en terreno (Soto et al. 1994). A los datos anteriores, se les agregó además la información de superficie, profundidad máxima, y biomasa zooplanctónica para cada sitio, descrita según Soto et al. (1994), y los datos de riqueza de especies zooplanctónicas, descrita según Soto \& De los Ríos (2006). A todo este conjunto de datos se les aplicó un análisis de componentes principales (PCA) utilizando el programa Xlstat, con el fin de determinar los potenciales factores que generen las diferencias posibles entre los sitios estudiados.

A los datos presencia y ausencia de especies según Soto \& De los Ríos (2006), se les aplicó el 


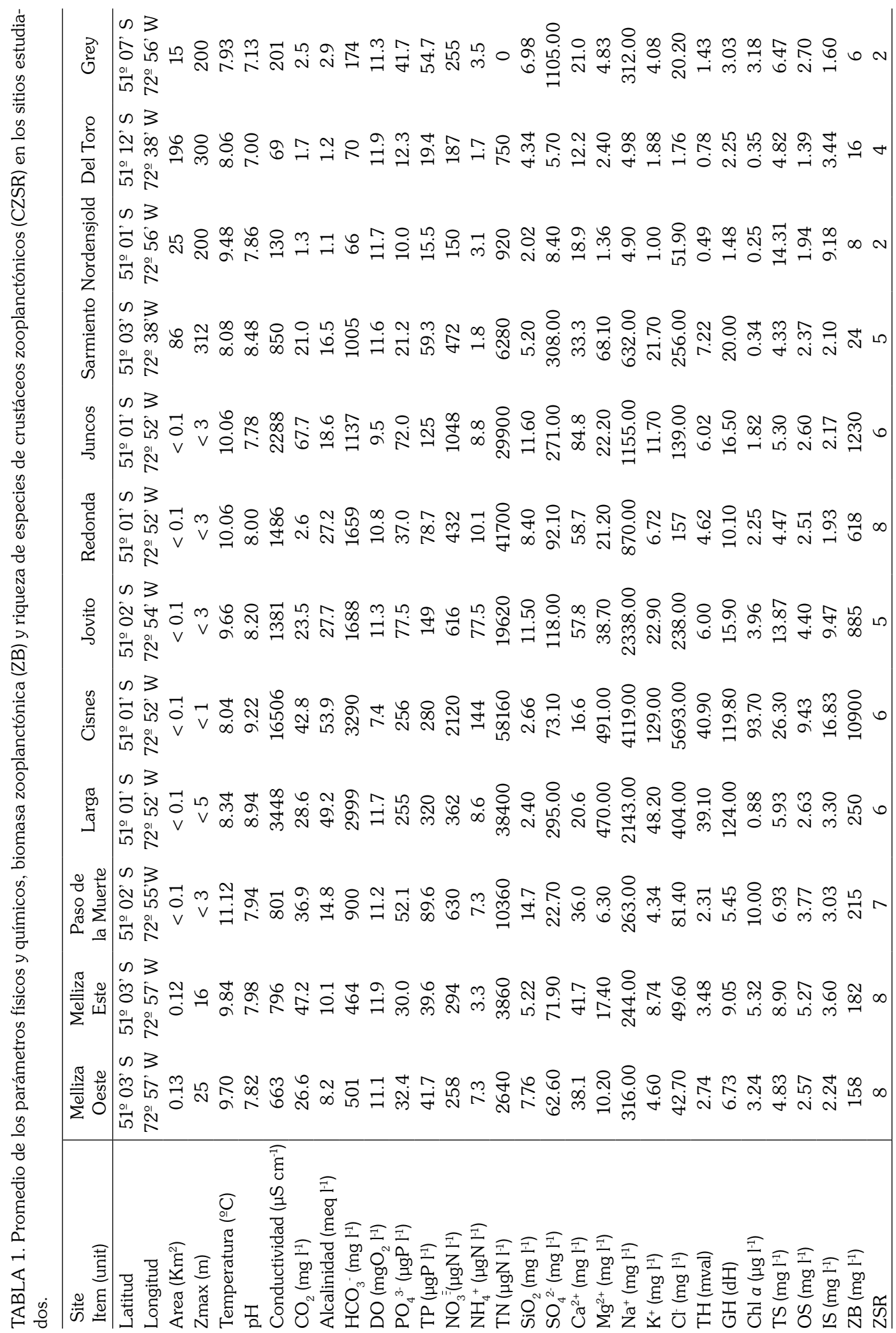


"índice de ponderación C" (Stone \& Roberts 1990), sobre la base que las asociaciones de especies serían aleatorias. Para esto se maneja una matriz de presencia y ausencia de especies (Gotelli 2000, Tondoh 2006, Tiho \& Johens 2007); en esta matriz se considera a las filas donde van las especies y los sitios que corresponden a las columnas, y sobre esta base se analizó según los siguientes modelos: (a) Modelo fijo-fijo: en este modelo las filas y columnas originales no son alteradas, y así cada simulación aleatoria contiene el mismo número de especies de la comunidad original (columna fija) y cada especie ocurre en la misma frecuencia que la comunidad original (fila fija). (b) Modelo fijo-equiprobable: en este modelo sólo la suma de las columnas se mantiene sin variación, y existe la misma posibilidad de encontrar especies por sitio (Gotelli 2000, Tiho \& Johens 2006). (c) Modelo fijo-proporcional: en este modelo la ocurrencia total de especies es mantenida como en la comunidad original, y la probabilidad de que alguna especie se presente en un sitio (columna) es proporcional al total de la columna por sitio (Gotelli 2000, Tiho \& Johens 2006, Tondoh 2007). Todos estos análisis se realizaron por medio del programa Ecosim versión 7.0 (Gotelli \& Entsminger 2001).

\section{RESULTADOS Y DISCUSIÓN}

Los resultados de la tabla 1, muestran que los lagos grandes tienden a ser oligotróficos, con bajas concentraciones de iones, baja biomasa zooplanctónica y baja riqueza de especies zooplanctónicas, mientras que los lagos chicos y las lagunas tienden a ser mesotróficos o eutróficos, con valores relativamente moderados en cuanto a sus concentraciones de iones y a la biomasa zooplanctónica y al número de especies zooplanctónicas. (Tabla 1). Estos resultados concuerdan con un primer estudio donde se consideró biomasa estatus trófico y biomasa zooplanctónica (Soto et al. 1994), donde se observó que los lagos patagónicos profundos donde tienen baja riqueza de especies asociado a condiciones de oligotrofia (Campos 1984, Soto \& Zúñiga 1991, Modenutti et al. 1998, Quiroz \& Drago 1999, Woelfl 2007). Esta condición de oligotrofia está acentuada además por los altos valores de la profundidad del epilimnion de estos lagos, lo que provoca una limitación por luz a la actividad fitoplanctónica (Soto 2002).
Los resultados muestran correlaciones directas significativas entre $\mathrm{pH}$, conductividad, alcalinidad, bicarbonato, fósforo reactivo soluble, nitrógeno en nitrato, amonio, nitrógeno total, $\mathrm{Mg}^{2+}, \mathrm{Na}^{+}, \mathrm{K}^{+}, \mathrm{Cl}^{-}$, dureza total, dureza alemana, clorofila "a", seston orgánico y biomasa zooplanctónica (Fig. 2a). Estos resultados se deberían presumiblemente a la alta evaporación a la que están expuestos estos ecosistemas debido a la exposición a los fuertes vientos, lo que repercute en las altas concentraciones iónicas y de nutrientes (Soto et al. 1994; Campos et al. 1994b, 1996). Por otro lado se observó una relación inversa entre profundidad máxima con $\mathrm{CO}_{2}$, nitrógeno total, riqueza de especies de crustáceos zooplanctónicos, $\mathrm{O}_{2}$ con fósforo reactivo soluble, nitrógeno en nitrato, nitrógeno total, amonio, $\mathrm{Na}^{+}$, $\mathrm{K}^{+}, \mathrm{Cl}^{-}$, clorofila "a", seston total, seston orgánico e inorgánico(Fig. 2a). Estos resultados serían similares a los descritos para grandes lagos de la Patagonia de Argentina (Quirós \& Drago 1999), los que por su gran volumen, y condición de baja o casi nula contaminación, presentan bajas concentraciones iónicas y de clorofila, mientras que la situación contraria sucede en lagunas pequeñas, debido al menor volumen de agua, donde hay más efecto por evaporación.

Los resultados del Análisis de Componentes Principales revelaron que las variables que contribuyeron con el 73,50\%, para el primer eje la contribución fue de 58,23\% y las variables importantes fueron $\mathrm{pH}$, conductividad, $\mathrm{CO}_{2}$, alcalinidad, nitrógeno total, $\mathrm{Mg}^{+2}, \mathrm{Na}^{+}, \mathrm{K}^{+}, \mathrm{Cl}^{\text {- }}$, dureza total, clorofila "a", seston (total, orgánico e inorgánico) y biomasa de especies de crustáceos zooplanctónicos (Fig. 2a). Mientras que para el segundo eje, la contribución fue de un $15,28 \%$, y las variables importantes fueron superficie, profundidad máxima, temperatura, $\mathrm{Si}, \mathrm{Ca}^{+} \mathrm{y}$ riqueza de especies zooplanctónicas (Fig. 2a). Estos resultados denotan que los parámetros químicos y las condiciones tróficas son más importantes que las características geográficas (área, profundidad máxima, temperatura) y riqueza de especies zooplanctónicas (Figs. 2a y 2b). Los resultados del Análisis de Componentes Principales, indican la existencia de dos grupos de lagos, el primero corresponde a lagos grandes profundos, oligotróficos, con bajas concentraciones minerales, baja biomasa y riqueza de especies de crustáceos zooplanctónicos (Tabla 1, Fig. 2b). El segundo grupo correspondió a lagos 

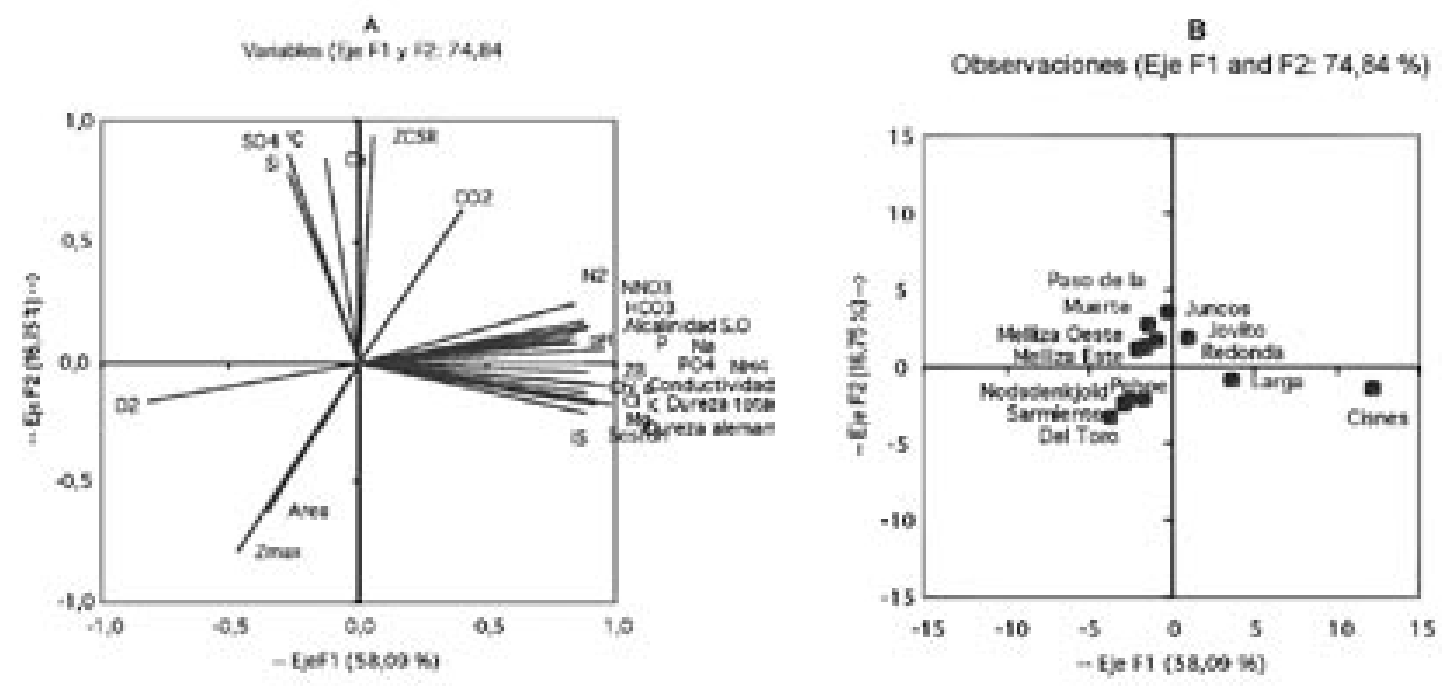

Fig. 2. Resultados de análisis de componentes principales para los sitios estudiados. A: contribución de las variables; B: localización de los sitios estudiados (1: Melliza Este; 2: Melliza Oeste;3: Cisnes; 4: Jovito; 5: Juncos; 6: Larga; 7: Paso de la Muerte; 8: Redonda; 9: Sarmiento; 10: Toro; 11: Norsdenkjold; 12: Grey)

pequeños y lagunas poco profundas, con conductividad moderada, mesotróficos, y biomasa y riqueza de especies crustáceos zooplanctónicos relativamente altas (Paso, Juncos, Melliza Este y Melliza Oeste; Tabla 1, Fig. 2b). Dentro de este grupo además se incluyen las lagunas Cisnes y Larga, que presentaron alta conductividad, altas concentraciones de nutrientes y de clorofila "a", alta biomasa de especies de crustáceos zooplanctónicos y baja diversidad de especies de crustáceos zooplanctónicos (Tabla 1, Fig. 2b). Por otro lado los resultados de la asociación de alta biomasa de crustáceos zooplanctónicos y alta concentración de clorofila y nutrientes concuerda con las observaciones de Samuelsson $(2003)^{1}$ y Burns \& Schallenberg (1998), quienes describen el rol de la disponibilidad de nutrientes como reguladores de tramas tróficas en lagos oligotróficos. Una situación totalmente inversa a la propuesta por ambos autores, sucedería en la Patagonia de Argentina, donde en condiciones de oligotrofia existiría baja actividad zooplanctónica (Balseiro et al. 2001, Modenutti et al. 2003), lo cual sería similar a lo observado en los grandes lagos estudiados en el presente trabajo (Tabla 1, Fig. 2b)

1 Samuelsson, K. 2003. Mechanisms estructuring the pelagic microbial food web. PhD Thesis, Department of Ecology and Environmental Sciences, Umea University, Sweden. $129 \mathrm{pp}$.
Un factor importante, en grandes lagos de la Patagonia es el efecto de la presencia de salmónidos asilvestrados (Soto et al. 1994, Soto et al. 2006, Soto et al. 2007, Becker et al. 2007, Pascual et al. 2007, Arismendi et al. 2009). Aunque en el presente estudio no se cuantificaron sus poblaciones, y no está demostrado un efecto potencial directo o indirecto de estas poblaciones de peces sobre zooplancton en lagos chilenos, es muy probable en sitios con poblaciones de salmónidos existan especies zooplanctónicas de menor tamaño corporal (Soto et al. 1994), por desaparición de grupos de gran tamaño como cladóceros y copépodos (Modenutti et al. 1998, Reissig et al. 2006). De igual modo existiría una serie de interacciones del tipo efecto cascada, donde los salmónidos podrían depredar sobre peces nativos o compartir sus nichos tróficos (Soto et al. 2007, Arismendi et al. 2009), lo cual no está del todo claro en cuanto a sus efectos sobre el zooplancton. Una situación diferente sucedería en presencia de peces nativos como puyes (Galaxias sp.), los cuales están presentes en las lagunas Melliza Este y Melliza Oeste (Soto et al. 1994), donde se observó que los peces nativos depredarían activamente sobre los copépodos calanoideos, favoreciendo la presencia de cladóceros. Finalmente en lagunas poco profundas (e.g.: Paso, Jovito, Redonda, Larga, Juncos y Cisnes), donde no se observaron peces, los altos valores de 
TABLA 2. Resultados del análisis de modelo nulo para los sitios estudiados, los valores de "P" inferiores a 0,05 indican que las asociaciones de especies son aleatorias.

$\begin{array}{lcccc}\text { Modelo } & \text { Índice observado } & \text { Índice Promedio } & \begin{array}{c}\text { Efecto Estándar } \\ \text { del Tamaño }\end{array} & \mathrm{P} \\ \text { Fijo-Fijo } & 5.000 & 4.527 & 5.624 & 0.000 \\ \text { Fijo-Proporcional } & 5.000 & 4.197 & 1.820 & 0.023 \\ \text { Fijo-Equiprobable } & 5.000 & 4.842 & 0.492 & 0.347\end{array}$

biomasa zooplanctónica y alto número de especies reportadas coincide con descripciones similares para sus contrapartes del sur de la Patagonia de Argentina (Reissig et al. 2006). No obstante, en estos sitios sin peces, las tramas tróficas se caracterizan por la presencia de la especie Parabroteas sarsi (Soto \& De los Ríos 2008), el cual es un activo depredador zooplanctónico, principalmente sobre copépodos juveniles y en menor grado sobre cladóceros (Modenutti et al. 2003, Reissig et al. 2004).

Los resultados de los modelos nulos denotan la ausencia de aleatorizaciones en las asociaciones de especies en dos (modelos fijo-fijo y fijo-proporcional) de las tres simulaciones, mientras que el modelo fijoequiprobable mostró presencia-ausencia de factores reguladores (Tabla 2). Estos resultados concuerdan con los obtenidos en el PCA, que indica la presencia de factores reguladores que afectarían la riqueza de especies de crustáceos zooplanctónicos, pero los resultados aparentemente contradictorios observados en el modelo fijo-equiprobable se deberían a la presencia de pocas especies que están presentes en casi la totalidad de los sitios estudiados, lo cual concuerda con otras descripciones similares para lagos chilenos (De los Ríos et al. 2008, De los Ríos 2008). Lamentablemente no hay más información publicada sobre el uso de estos modelos en ecología lacustre; no obstante, sobre la base de las referencias disponibles, se podría indicar que el uso de modelos nulos basados en presencia y ausencia de especies en lagos chilenos, requeriría de su aplicación con precauciones, considerando la baja riqueza de especies y presencia de pocas especies muy frecuentes en la mayoría de los sitios.

\section{AGRADECIMIENTOS}

Los resultados del presente trabajo en terreno fueron financiados por el proyecto FONDECYT 81788, se agradece la participación de Luis Zúñiga y de la Dirección General de Investigación de la Universidad
Católica de Temuco. Esta publicación está dedicada a la memoria de Hugo Campos (fallecido en 1998) como pionero de la limnología chilena.

\section{LITERATURA CITADA}

Arismendi, I., D. Soto, B. Penaluna, C. Jara, C. Leal \& J. León-Muñoz 2009. Aquaculture, non-native salmonid invasions and associated declines of native fishes in Northern Patagonian lakes. Freshwater Biology 54: 1154-1147.

Balseiro, E.G., B.E. Modenutti \& C.P. Queimaliños 2001. Feeding of Boeckella gracilipes (Copepoda, Calanoida) on ciliates and phytoflagellates in an ultraoligotrophic Andean lake. Journal of Plankton Research 23: 849-857.

Becker, L.A., M.A. Pascual \& N.G. Basso 2006. Colonization of the southern Patagonia Ocean by exotic Chinook salmon. Conservation Biology 21: 1347-1352.

Burns C.W. \& M. Schallenberg 1998. Impacts of nutrients and zooplankton on the microbial food web of an ultra-oligotrophic lake. Journal of Plankton Research 20:1501-1525

Campos, H. 1984. Limnological studies of Araucanian lakes (Chile) Verhandlungen International Verienüng fur Angewaldte und Applied Limnolologie 22:1319-1327.

Campos, H., D. Soto, W. Steffen, G. Agüero, O. Parra \& L. Zúñiga 1996. Limnological studies of Amarga Lagoon, Chile: a saline lake in Patagonian South America. International Journal of Salt Lake Research4:301-314.

Campos, H., D. Soto, W. Steffen, G. Agüero, O. Parra \& L. Zúñiga 1994a. Limnological studies of Lake del Toro (Chile) morphometry, physics, chemistry and plankton. Archiv für Hydrobiologie Supplement 99:199-215.

Campos, H., D. Soto, W. Steffen, G. Agüero, O. Parra \& L. Zúñiga 1994b. Limnological stu- 
dies of Lake Sarmiento (Chile): A subsaline lake from Chilean Patagonian. Archiv für Hydrobiologie Supplement 99:217-234.

De los Ríos, P., N. Rivera \& M. Galindo 2008. The use of null models to explain crustacean zooplancton associations in shallow water bodies of the Magellan region, Chile. Crustaceana 81: $1219-1128$

De los Ríos, P. 2008. A null model for explain crustacean zooplancton species associations in central and southern Patagonian inland waters. Anales Instituto Patagonia (Chile) 36: 25-33.

De los Ríos, P. \& D. Soto 2007. Crustacean (Copepoda and Cladocera) zooplankton richness in Chilean Patagonian lakes. Crustaceana 80: 285-296.

De los Ríos, P. \& D. Soto 2006. Structure of the zooplanktonic crustaceous Chilean lacustre assamblages: role of the trophic status and protection resources. Crustaceana 79: 2332.

De los Ríos, P. 2005. Richness and distribution of zooplanktonic crustacean species in Chilean altiplanic and southern Patagonia ponds. Polish Journal of Environmental Studies 14: 817-822

Gillooly, J.F. \& S.I. Dodson 2000. Latitudinal patterns in the size distribution and seasonal dynamics of new world, freshwater cladocerans. Limnology \& Oceanography 45: 22-30.

Gotelli, N.J. 2000. Null models of species co-occurrence patterns. Ecology, 81: 2606-2621.

Gotelli, N.J. \& G.L. Entsminger 2001. EcoSim: Null models software for ecology. Version 7. Acquired Intelligence Inc. \& Kesey-Bear. Jericho, VT 05465. http://garyentsminger. com/ecosim.htm.

Jaksic, F. 2001. Ecología de comunidades. Ediciones Pontificia Universidad Católica de Chile, Santiago de Chile. 233 pp.

Modenutti, B.E., E.G. Balseiro, C.P. Queimaliños, D.A. Suárez, M.C. Diéguez \& R.J. Albariño 1998. Structure and dynamics of food webs in Andean lakes. Lakes and Reservoirs, Research and its Management 3: 179-186.

Modenutti, B.E., C. Queimaliños, E. Balseiro \& M. Reissig 2003. Impacts of different zooplankton structures on the microbial food web of a South
Andean oligotrophic lake. Acta Oecologica 24: 289-298.

Pascual, M.A., V. Cussac, B. Dyer, D. Soto, P. Vigliano, S. Ortubay \& P. Macchi 2007. Freshwater fishes of Patagonia in the 21st Century after hundred years of human settlement, species introduction and environmental change. Aquatic Ecosystem Health and Management 10(2): 212-227.

Quirós, R. \& E. Drago 1999. The environmental state of Argentinean lakes: an overview. Lakes and Reservoirs, Research and its Management.4:55-64

Reissig, M., C. Trochine, C. Queimaliños, E. Balseiro \& B. Modenutti 2006. Impacts of fish introduction on planktonic food webs in lakes of the Patagonian Plateau. Biological Conservation, 132: 437-447.

Reissig, M., B. Modenutti, E. Balseiro \& C. Queimaliños 2004. The role of predaceous copepod Parabroteas sarsi in the pelagic food web of a large deep Andean lake. Hydrobiologia 524: 67-77.

Soto, D., I. Arismendi, C. Di Prinzio \& F. Jara 2007. Establishment of Chinook salmon (Oncorhynchus tshawytscha) in Pacific basins of southern South America and its potential ecosystem implications. Revista Chilena de Historia Natural, 80: 81-98.

Soto, D., I. Arismendi, J. González, J. Sanzana, F. Jara, C. Jara, E. Guzmán \& A. Lara 2006. Southern Chile, trout and salmon country: invasion patterns and threat for native species. Revista Chilena de Historia Natural, 79: 97-117.

Soto, D. 2002. Oligotrophic patterns in southern Chile lakes: the relevance of nutrients and mixing depth. Revista Chilena de Historia Natural 75: 377-393.

Soto, D., H. Campos, W. Steffen, O. Parra \& L. Zúñiga 1994. The Torres del Paine lake district (Chilean Patagonia): A case of potentially N-limited lakes and ponds. Archiv für Hydrobiologie 99:181-197.

Soto, D., \& P. De los Ríos 2006. Trophic status and conductivity as a regulators in daphnid dominance and zooplankton assemblages in lakes and ponds of Torres del Paine National Park. Biologia, Bratislava 61:541-546. 
Soto, D. \& L. Zúñiga 1991. Zooplankton assemblages of Chilean temperate lakes: a comparison with North American counterparts. Revista Chilena de Historia Natural 64: 569-581.

Tiho, S. \& J. Johens 2007. Co-occurrence of earthworms in urban surroundings: a null models of community structure. European Journal of Soil Biology 43: 84-90.

Tondoh, J.E. 2006. Seasonal changes in earthworm diversity and community structure in central
Côte d'Ivoire. European Journal of Soil Biology 42: 334-340.

Williams, W.D. 1998 Salinity as a determinant of the structure of biological communities in salt lakes. Hydrobiologia 381:191-201,

Woelfl, S. 2007. The distribution of large mixotrophic ciliates (Stentor) in deep North Patagonian lakes (Chile): First results. Limnologica 37:28-36. 
P. DE LOS RÍOS \& D. SOTO 